\title{
Downlink Performance of Cooperative Distributed Antenna Systems over Nakagami- $m$ Fading Channels in Multi-cell Environment
}

\author{
Yuxi Liu*, Lina Zheng ${ }^{\dagger}$, He Chen ${ }^{\ddagger}$, and JiaKui Zhao* \\ * State Grid Electric Power Research Institute \\ Beijing, China 100192 \\ Email: liouyuxisimon@163.com,jkzhao@gmail.com \\ ${ }^{\dagger}$ School of Information Science and Engineering \\ Shandong University, Jinan, China 250100 \\ Email: zhenglina@sdu.edu.cn \\ $\ddagger$ School of Electrical and Information Engineering \\ The University of Sydney, NSW, Australia, 2006 \\ Email: chlyy2005@yahoo.cn
}

\begin{abstract}
In this paper, we investigate the downlink performance of cooperative distributed antenna systems (DAS) in multicell environment, including ergodic capacity, outage probability, and bit error rate (BER). Blanket transmission, all distributed antenna modules (DAMs) in one cell are utilized to transmit signals, is considered and can be seemed as virtual multipleinput single-output (V-MISO) system. The interference plus noise is considered as Gaussian noise with fixed variance by central limit theorem (CLT), while the channels are assumed to suffer from independent and identical Nakagami- $m$ fading together with propagation path loss. From the perspective of information theory, the closed-form expressions for ergodic capacity and BER are derived via iterative method, while for outage probability, it is given through algebraic operations. Finally extensive simulations are performed to validate our theoretical analysis.
\end{abstract}

Keywords-Distributed antenna systems (DAS), central limit theorem (CLT), Nakagami- $m$ fading, blanket transmission.

\section{INTRODUCTION}

The rapid growth in mobile communications has led to an increasing demand for much higher data rate than can be provided today. Many advanced techniques, such as distributed antenna systems (DAS), cooperative communication, and beamforming, are proposed to overcome the aforementioned problem nowadays.

Originally, DAS were introduced to cover the dead spots in indoor wireless communications [1]. Then, it was exploited to provide better coverage and increase battery life coupled with system capacity [2-3]. Different from the conventional cellular network that the transmit modules centralize at a location, the antenna modules (AMs) of DAS are geographically distributed to reduce access distance. Within a reference cell, AMs are connected to a home base station (BS) or central unit (CU) through dedicated wires, high-speed optical fiber backbone, or an exclusive RF link, which are high capacity and low latency and allow a reliably fast information exchange among them. This configuration is considered as a virtual multiple-input single-output (V-MISO) system that can achieve diversity gain.

In [4], Zhou et al, gave an overview of the distributed wireless communication system (DWCS), drew a comparison with traditional cellular networks, and discussed several primary problems. The concept of virtual cell was introduced in [5], where analytical outage probability expressions were derived. Moreover, the uplink capacity in multi-cell environment was further investigated in [6]. However, neither of them involve the downlink scenario duo to its complexity. Without considering the multi-cell situation, information-theoretic capacity of MIMO DAS has been studied by Xiao et al in [7]. Besides, considering the downlink channels undergo Rayleigh fading, Choi has formulated the closed-form expressions for ergodic capacity and outage probability of DAS in [2][8].

Specially, cooperation is another effective method to provide higher transmission rates and improve robustness to channel fading. It can allow the mobile terminals (MTs) or base stations (BSs) with single antenna to reap the benefits of multi-input multi-output (MIMO) systems [7]. Cooperative communication as a new class of methods has been put forward, and enables single antenna mobile terminals to share their antennas and generate a virtual multiple antenna systems to obtain diversity gain [9]. Base station cooperation (BSC), the configuration of which is consistent with DAS, is one branch of cooperative communication [10]. With respect to the aforementioned cooperation and DAS, You et al, give a conceptual description about the cooperative DAS along with key techniques [11]. The multi-cell processing capacity of the cellular MIMO uplink channel in correlated Rayleigh fading environment has been analyzed and its principle is similar to DAS [12].

Nakagami- $m$ distribution has drawn special attention for its convenient manipulation and wide applicability. It is assumed to be composed of cluster of multipath waves with no dominant components within any cluster. Rayleigh distribution, Rice distribution and uniform distribution are the special cases of Nakagami- $m$ distribution [13-14]. To the best of our knowledge, there are few literatures to analyze the system performance of DAS suffering from Nakagami- $m$ fading.

In this paper, we focus on the performance analysis of the cooperative DAS over Nakagami- $m$ fading channel in multicell environment. The configuration of DAS is consistent with the deployment of base station cooperation within the refer- 
ence cell. The closed-form expressions for ergodic capacity and bit error rate (BER) in the downlink DAS are derived via iterative method, while the outage probability is given through algebraic operations.

The rest of the paper is organized as follows. Section II describes the system model. The system performance of DAS including achievable capacity, outage probability and average BER is analyzed in Section III. Some practical concerns are discussed and numerical results as well as discussion are provided in Section IV. Finally, Section V concludes this paper.

Notation: Throughout this paper, E [.] denotes the expectation, $(\cdot)^{\prime}$ represents differentiation and $f_{X}(x)$ is the PDF of random variable $X$.

\section{System And Channel Model}

In DAS, the main processing modules are centralized at a central unit (CU) and connected with the distributed antenna modules (DAMs) [2-3]. Adopting the Wyner's planar cellular pattern [15-16], where each cell "sees" the signal radiated in the adjacent cell cites, we draw the configuration of cooperative DAS in Fig. 1. It is assumed that each cell is covered by a small home base station and distributed antenna modules, and the actual number of DAMs is determined by coverage, user densities as well as other environments factors. For simplicity, six DAMs in one cell are taken into account as a reasonable example in the paper. We arbitrarily choose one cell as research object indexed by $\operatorname{Cell}_{j}(j=0)$ and denote the adjacent six cells as $\operatorname{Cell}_{j}(j=1, \cdots, 6)$. The home base station is indexed as $R_{0}$, and the six DAMs are denoted by $R_{\mathrm{i}}(i=1, \cdots, 6)$ within each cell. Similar to the configuration of BSC, the six DAMs as well as the home base station are connected to the $\mathrm{CU}$. The total transmit power of the $i$ th DAM in the $j$ th cell is denoted by $P_{i}^{(j)}$, while the total transmit power of each cell is $\sum_{i=0}^{6} P_{i}^{(j)}=P$. The normalized distances between MT and the home base station as well as between the mobile terminal (MT) and the DAMs in the $j$ th cell are denoted by $d_{i}^{(j)}(i \in 0, \cdots, 6)$, so the corresponding propagation pathloss has the following equation $L_{i}^{(j)}=\left(d_{i}^{(j)}\right)^{-\sigma}$, where $\sigma$ is the pathloss exponent.

Similar to [2], it is assumed that the MT moves away from the home base station to the worst case position (W) on cellboundary, which is depicted in Fig. 1. We assume one MT is active in each cell which can be realized by utilizing timedivision multiple-access (TDMA) technique, and the channel suffers from block-fading, namely, the fading coefficients of the channel remain invariant in a packet and vary from packet to packet.

In each packet, the discrete time baseband signal received by the MT can be written as (discrete-time dependence is omitted for simplicity of notation):

$$
y=\mathbf{h}^{(0)} \mathbf{x}^{(0)}+\sum_{i=1}^{6} \mathbf{h}^{(i)} \mathbf{x}^{(i)}+n
$$

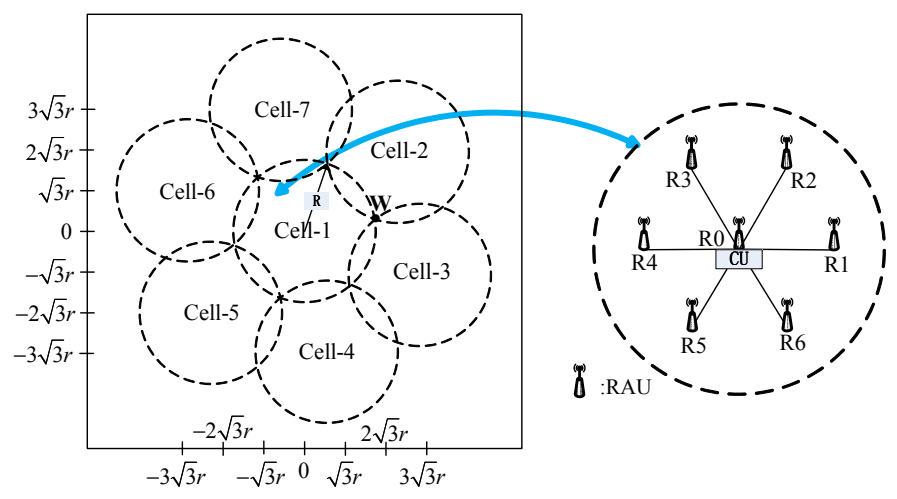

Fig. 1. Planar Wyner DAS Model.

where $\mathbf{h}^{(j)}=\left[\sqrt{L_{0}^{(j)}} h_{0}^{(j)}, \sqrt{L_{1}^{(j)}} h_{1}^{(j)}, \cdots, \sqrt{L_{6}^{(j)}} h_{6}^{(j)}\right]$ is the channel vector in the $j$ th cell. $h_{i}^{(j)}$ denotes short term fading from the $i$ th distributed antenna module in the $j$ th cell to the active MT and is an independent identical Nakagami$m$ random variable with zero mean and variance $\Omega$, while $L_{i}^{(j)}$ is the corresponding propagation pathloss. $\mathbf{x}^{(j)}=$ $\left[x_{0}^{(j)}, x_{1}^{(j)}, \cdots, x_{6}^{(j)}\right]^{T}$ denotes the transmit signal vector in the $j$ th cell, where $x_{i}^{(j)}$ is the transmit signal of the $i$ th DAM in the $j$ th cell with power $P_{i}^{(j)}=\mathrm{E}\left[\left|x_{i}^{(j)}\right|^{2}\right] \cdot n$ is the additive Gaussian noise with variance $\mathrm{E}\left[n n^{H}\right]=\sigma_{n}^{2}$, which is assumed to be independent of the transmit signal $x_{i}^{(j)}$.

The probability density function (PDF) of $h_{i}^{(j)}$ is given as

$$
f_{\left|h_{i}^{(0)}\right|}(h)=\frac{2}{\Gamma(m)}\left(\frac{m}{\Omega}\right)^{m} h^{2 m-1} \exp \left(-m h^{2} / \Omega\right)
$$

where $\Omega=\mathrm{E}\left(\left|h_{i}^{(0)}\right|^{2}\right), m=\frac{\mathrm{E}\left(\left|h_{i}^{(0)}\right|^{2}\right)}{\operatorname{Var}\left(\left|h_{i}^{(0)}\right|^{2}\right)}$ is the fading severity parameter, and $\Gamma(\cdot)$ denotes the Euler Gamma function.

Note that $f_{\left|h_{i}^{(0)}\right|}(h)$ reduces to the Rayleigh distribution for $m=1$, to the uniform distribution on the circle of radius $\sqrt{\Omega}$ for $m \rightarrow \infty$, and for $m=0.5$, (2) gives the one-sided Gaussian PDF. In the paper, we assume $m$ is integer and the interference plus noise is a complex Gaussian random variable $z$ with variance $\delta_{z}^{2}=\sum_{i=1}^{6} \sum_{j=0}^{6} L_{j}^{(i)} P_{j}^{(i)} \cdot \Omega+\sigma_{n}^{2}$ by central limit theorem (CLT).

\section{Performance Analysis}

It is assumed that the channel state information (CSI) is known at the receiver and the channel is ergodic.

The received signal at MT has been given in (1) and the useful signal can be rewritten as

$$
z=\left[\mathbf{h}^{(0)}\right] \mathrm{E}\left[\left(\mathbf{x}^{(0)}\right)\left(\mathbf{x}^{(0)}\right)^{\mathrm{H}}\right]\left[\mathbf{h}^{(0)}\right]^{\mathrm{H}}=\sum_{i=0}^{6} \gamma_{i}^{(0)}
$$


where $\gamma_{i}^{(0)}=\left|h_{i}^{(0)}\right|^{2} L_{i}^{(0)} P_{i}^{(0)}=a_{i}^{(0)} \cdot\left|h_{i}^{(0)}\right|^{2}$, and $\left|h_{i}^{(0)}\right|$ submits to Nakagami- $m$ distribution. So the PDF of $\gamma_{i}^{(0)}$ is given by:

$$
f_{\gamma_{i}^{(0)}}(\gamma)=\frac{1}{\Gamma(m)}\left(\frac{m}{\Omega a_{i}^{(0)}}\right)^{m} \gamma^{m-1} \exp \left(-m \gamma / \Omega a_{i}^{(0)}\right)
$$

Let $\frac{m}{\Omega a_{i}^{(0)}}=b_{i}^{(0)},(4)$ can be reduced to

$$
f_{\gamma_{i}^{(0)}}(\gamma)=\frac{1}{\Gamma(m)}\left(b_{i}^{(0)}\right)^{m} \cdot \gamma^{m-1} \cdot \exp \left(-b_{i}^{(0)} \gamma\right)
$$

The moment generate function (MGF) of $f_{\gamma_{i}^{(0)}}(\gamma)$ in (5) is given as:

$$
F_{\gamma_{i}^{(0)}}(s)=\left[\frac{b_{i}^{(0)}}{s+b_{i}^{(0)}}\right]^{m}
$$

Referring to [17], the MGF of (3) can be written in the following equation.

$$
F_{z}(s)=\prod_{i=0}^{6} F_{\gamma_{i}^{(0)}}(s)=\sum_{i=0}^{6} \sum_{p=1}^{m} \frac{n_{i p}}{\left(s+b_{i}^{(0)}\right)^{p}}
$$

where

$$
n_{i p}=\frac{1}{(m-p) !} \frac{\mathrm{d}^{m-p}}{\mathrm{~d} s^{m-p}}\left[\left(s+b_{i}^{(0)}\right)^{m} F_{z}(s)\right]_{s=-b_{i}^{(0)}}
$$

Applying inverse Laplace transform to $F_{z}(s)$, the PDF of $\mathrm{z}$ is given as:

$$
f(z)=\sum_{i=0}^{6} \sum_{p=1}^{m} \frac{n_{i p}}{(p-1) !} z^{p-1} \exp \left(-b_{i}^{(0)} z\right) \quad z>0
$$

According to mathematical statistics, the PDF of $\gamma=\frac{z}{\delta_{z}^{2}}$ can be written as:

$$
f_{\gamma}(\gamma)=\sum_{i=0}^{6} \sum_{p=1}^{m} \frac{n_{i p}}{(p-1) !}\left(\delta_{z}^{2}\right)^{p} \gamma^{p-1} \exp \left(-b_{i}^{(0)} \delta_{z}^{2} \gamma\right)
$$

\section{A. Ergodic Capacity}

The ergodic capacity of the target MT at a given location can be achieved by

$$
\begin{aligned}
C_{e} & =\mathrm{E}_{\mathrm{h}}\left[\log _{2}(1+\gamma)\right] \\
& =\int_{\gamma=0}^{\infty} \log _{2}(1+\gamma) f_{\gamma}(\gamma) d \gamma \\
& =\frac{1}{\ln 2} \sum_{i=0}^{6} \sum_{p=1}^{m} \frac{n_{i p}\left(\delta_{z}^{2}\right)^{p}}{(p-1) !} \cdot \Phi_{p}
\end{aligned}
$$

where

$$
\begin{aligned}
\Phi_{p}= & \frac{1}{b_{i}^{(0)} \delta_{z}^{2}}\left[\sum_{l=0}^{p-1}(-1)^{p-1-l}\left(\begin{array}{c}
p-1 \\
l
\end{array}\right)\left(b_{i}^{(0)} \delta_{z}^{2}\right)^{-l}\right. \\
& \left.\times \exp \left(b_{i}^{(0)} \delta_{z}^{2}\right) \Gamma\left(l, b_{i}^{(0)} \delta_{z}^{2}\right)+(p-1) \Phi_{p-1}\right]
\end{aligned}
$$

with $\left(\begin{array}{c}p-1 \\ l\end{array}\right)=\frac{p !}{n !(p-n) !}$ and $\Gamma(\alpha, x)=\int_{x}^{\infty} e^{-t} t^{\alpha-1} d t$ representing the incomplete Gamma function defined in [18]. For continuity, we set $\Phi_{0}=1$.

\section{B. Outage Probability}

In this section, the closed-form expression for the outage probability is derived. In DAS, if the capacity of a MT is less than the threshold value $R$, the quality of service (QoS) of the cellular network is not satisfied.

According to $C=\log _{2}(1+\gamma)$ and (10), the outage probability of the MT at a given location is derived as:

$$
\begin{aligned}
& P_{\text {out }}[C<R]=\operatorname{Pr}\left[\gamma<2^{R}-1\right] \\
& =\int_{0}^{2^{R}-1} \sum_{i=0}^{6} \sum_{p=1}^{m} \frac{n_{i p}}{(p-1) !}\left(\delta_{z}^{2}\right)^{p} \gamma^{p-1} \exp \left(-b_{i}^{(0)} \delta_{z}^{2} \gamma\right) d \gamma \\
& =\sum_{i=0}^{6} \sum_{p=1}^{m} \frac{n_{i p}}{(p-1) !}\left(\delta_{z}^{2}\right)^{p} \underbrace{\int_{0}^{2^{R}-1} \gamma^{p-1} \exp \left(-b_{i}^{(0)} \delta_{z}^{2} \gamma\right) d \gamma}_{\Upsilon_{1}}
\end{aligned}
$$

where $R$ is the threshold guaranteeing a desired QoS, and

$$
\Upsilon_{1}=\left(b_{i}^{(0)} \delta_{z}^{2}\right)^{-p} \gamma\left(p,\left(2^{R}-1\right) b_{i}^{(0)} \delta_{z}^{2}\right)
$$

where $\gamma(\alpha, x)=\int_{0}^{x} e^{-t} t^{\alpha-1} d t$ is the incomplete Gamma function [18].

Therefore, the closed-form expression of (13) can be formulated as:

$$
P_{\text {out }}[C<R]=\sum_{i=0}^{6} \sum_{p=1}^{m} \frac{n_{i p}}{(p-1) !}\left(\delta_{z}^{2}\right)^{p} \Upsilon_{1}
$$

\section{Average BER Analysis}

We assume all the transmit modules in multi-cell environment adopt the same modulation (BPSK, QPSK, or $M$ PSK). The closed-form expression of BER is derived in the following. For different modulation formats used in wireless communication, the system average BER is expressed as

$$
P_{B E R}=\alpha E[\operatorname{erfc}(\sqrt{\beta \gamma})]
$$

where $\alpha, \beta>0, \operatorname{erfc}(\mathrm{x})=\frac{2}{\sqrt{\pi}} \int_{\mathrm{x}}^{\infty} \exp \left(-\mathrm{t}^{2}\right) \mathrm{dt}$, and $(\alpha, \beta)$ are constants depending on the type of modulation. Noting that for $\alpha=0.5, \beta=1$ and $\alpha=1, \beta=1$, we obtain the well known probability of exact binary phase shift keying (BPSK) and exact quadrature phase shift keying (QPSK) transmission over Nakagami- $m$ fading channel respectively. For $M$-PSK 
$\alpha=1 /\left(2 \log _{2} M\right), \beta=\frac{1}{2} \log _{2} M \times \sin ^{2} \pi / M$ can be used to approximate the BER [19-20].

The average BER can be derived as follows:

$$
\begin{aligned}
P_{B E R}(e) & =\int_{0}^{\infty} P(e \mid \gamma) f_{\gamma}(\gamma) d \gamma \\
& =\sum_{i=0}^{6} \sum_{p=1}^{m} \frac{\alpha \cdot n_{i p}\left(\delta_{z}^{2}\right)^{p}}{(p-1) !} \Psi_{p}
\end{aligned}
$$

where $\Psi_{p}=\frac{\beta^{1-p}}{b_{i}^{(0)} \delta_{z}^{2}}\left[(p-1) \Psi_{p-1}-\frac{1}{\sqrt{\pi}} \cdot \frac{\Gamma\left(p-\frac{1}{2}\right)}{\left(1+\frac{b_{i}^{(0)} \delta_{z}^{2}}{\beta}\right)^{p-\frac{1}{2}}}\right]$

Similar to the setting of $\Phi_{0}$, we also set $\Psi_{0}=1$ for continuity.

\section{Simulation Results And Discussion}

To interpret the information theoretic results for DAS in Nakagami- $m$ fading channels, it is critical to establish the relation of the various system modeling parameters with real-word scenarios. Throughout the paper, the simulation parameters are set as follows. The transmit power of the home base station is $0.4 P$, while each DAM is $0.1 P$. It is assumed that $P=10 \mathrm{~dB}$, $\sigma_{n}^{2}=1, \sigma=4.0$, and $0<d \leq 1$. In simulation, all the home BSs as well as DAMs in Fig. 1 adopt BPSK modulation.

Fig. 2 displays that with the increase of $m$, the capacity of MT becomes higher, but as $m$ is larger than 5 , the variations are not obvious, namely, the curves almost superpose. This is because when the channels undergo Nakagami- $m$ distribution and $m$ becomes larger, the differences among Nakagami- $m$ ( $m=6, \cdots, N)$ distributions turn to be weakness (refer to (2)). In Fig. 2, when MT moves to $d=0.6$, the capacity is superior to surroundings, since in this location, there exits one DAM within the cell that dominates the transmit signal, as shown in Fig. 1. When MT locates at the cell boundary, the interference from adjacent cells plays a predominant part in signal-to-interference-plus-noise-ratio (SINR), so the capacity cuts down.

In Fig. 3, when MT reaches at the range $d \in(0,0.75)$, the larger is $m$, the lower is the outage probability, while $d \in$ $(0.75,1)$, it is inverse. The reason is that in $d \in(0.75,1)$, the signal of $m=1$ is smaller than $m=3$ or $m=5$, meanwhile the interference from adjacent cells is also smaller than $m=$ 3 or $m=5$. But at the cell boundary, the interference has greater impact on the received signal than the useful signal, so SINR of $m=1$ is higher than that of $m=3$ or $m=5$. In $d \in(0,0.4)$ and $d \in(0.6,1)$, the curves are monotonically increasing, since when $d \in(0,0.4)$ the home base station as well as the corresponding DAMs dominates the signal, and when $d \in(0.6,1)$ the interference from the adjacent cells become dominant. As $d \in(0.4,0.6)$, the curve is decreasing, for the dominant signal becoming weak and interference plus noise being strengthen.

Fig. 4 depicts the capacity versus the pathloss exponent $\sigma$ at $d=0.6$. Shown in Fig. 4, the capacity of MT becomes

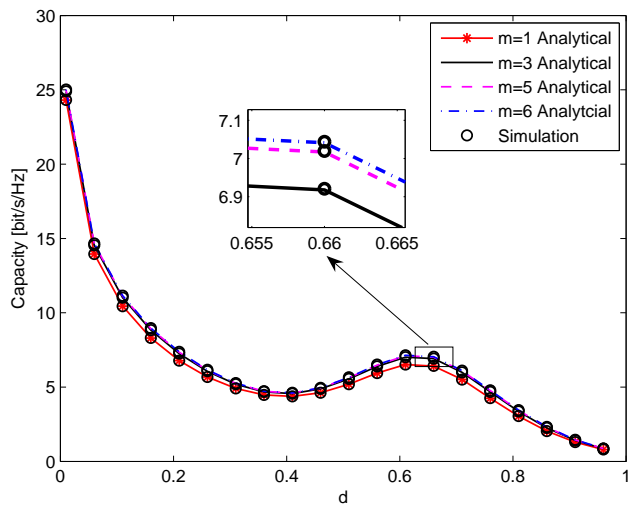

Fig. 2. Ergodic capacity with CSIR versus the normalized distance from the home base station to MT $(\Omega=1)$.

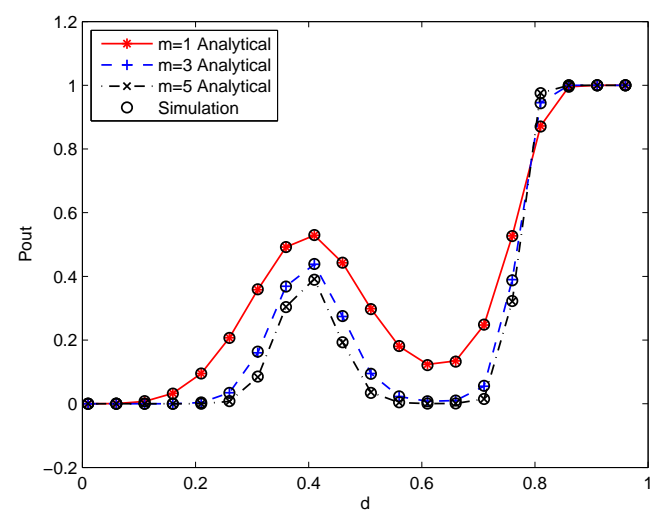

Fig. 3. Outage probability vs the normalized distance from the home base station $(\Omega=1)$.

higher as $m$ increases. When $m \geq 3$, the distinction of the curves is diminishing. This is because as given in (11), the capacity will become lager with the rise of $m$; otherwise, its result will not change in case $m$ reaches some threshold. So, in the paper, the chosen of $\sigma=4.0$ is reasonable.

The BER of the active MT is described in Fig. 5. As $m$ turns to be larger, the performance becomes better. When $d \in(0,0.4)$ the BER curve is increasing, because when MT moves away from the home base station, the strength of the received signal drops of gradually, while in $d \in(0.4,0.6)$, the curve is decreasing, for one of the DAMs within the reference cell becoming dominant. The curve is also decreasing in $d \in(0.6,1)$, as the interference from adjacent cells has greater effects on the SINR than the useful signal. All of the interpretations are consistent with that of the Fig. 2 as well as Fig. 3.

\section{Conclusions}

In the paper, we derive the closed-form expressions of ergodic capacity, outage probability as well as bit error rate over Nakagami- $m$ fading channels. When $m=1$, it is Rayleigh fading, which is consistent with Choi. As $m$ changes 


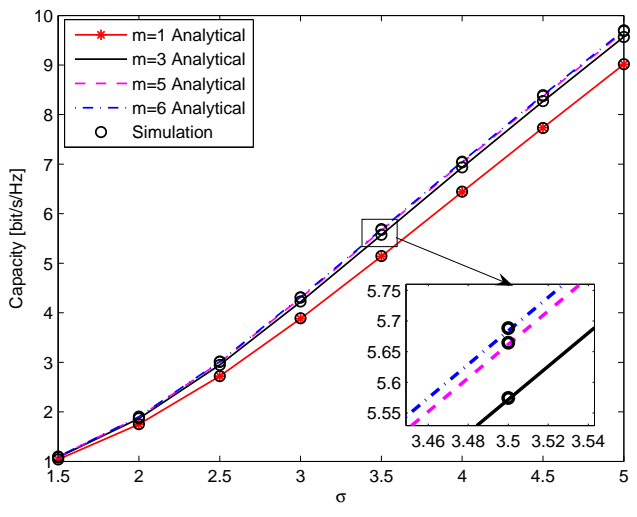

Fig. 4. Capacity versus the pathloss exponent $(\Omega=1), d=0.6$.

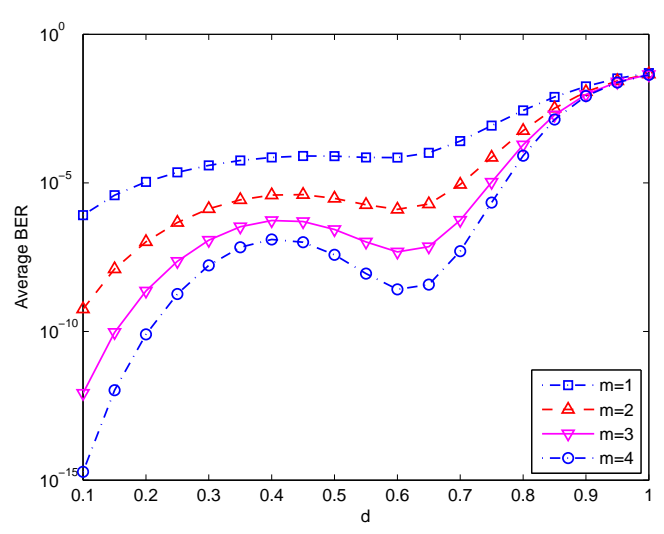

Fig. 5. Average BER versus the normalized distance from the home base station $(\Omega=1$, BPSK).

to be larger, the capacity of the MT becomes higher, the outage probability of the MT is lower, and the BER of the MT turns to be better, but the system performance changes very little while $m$ reaches a certain value. The simulations results demonstrate the correctness and efficiency of the analysis.

\section{ACKNOWLEDGMENT}

This work was supported by the Science and Technology Project of SGEPRI (State Grid Electric Power Research Institute) entitled "Research on the Information Processing and Typical Applications for Electric Internet of Things", the Science and Technology Projects of State Grid Corporation of China entitled "Research on the Integrated Supporting Technologies for Intelligent Marketing Business based on the International IEC-CIM/CIS Standard", "Research on the Technologies of Analysis and Pre-Warning for Company Operation", and Independent Innovation Foundation of Shandong University (2010JC007).

\section{REFERENCES}

[1] A. A. M. Saleh, A. J. Rustako, and R. S. Roman, "Distributed antennas for indoor radio communications," IEEE Trans. Commun., vol. 35, no. 12, pp. 1245-1251, Dec. 1987.
[2] Wan Choi and J.G. Andrews, "Downlink performance and capacity of distributed antenna systems in a multicell environment," IEEE Trans. Wireless Commun, vol. 6, no. 1, 2007.

[3] D. Castanheira, and A. Gameiro, "Distributed Antenna System Capacity Scaling," IEEE Wireless Commun., vol. 17, no. 3, pp.68-75, June, 2010.

[4] S. Zhou, M. Zhao, X. Xu, and Y. Yao, "Distributed wireless communication systems: A new architecture for future public wireless access," IEEE Commmu. Mag., vol. 41, no. 3, pp.108-113, Mar., 2003.

[5] L. Dai, S. Zhou, and Y. Yao, "Capacity analysis in CDMA distributed antenna systems," IEEE Trans. Wireless Commun., vol. 4, no. 6, pp. 2613-2620, Nov. 2006.

[6] E. Katranaras, M. A. Imran, and C. Tzaras, "Uplink capacity of a variable density cellular system with multicell processing," IEEE Trans. Commun., vol. 57, no. 7, pp.2098-2108, July, 2009.

[7] L. Xiao, L. Dai, H. Zhang, S. Zhou, and Y. Yao, "Information-theoretic capacity analysis in MIMO distributed antenna systems," in Proc. IEEE Veh. Technol. Conf., pp. 779-782, Apr. 2003.

[8] Wan Choi and J.G. Andrews, "Theoretical limits of cellular systems with distributed antennas," in Distributed Antenna Systems: Open Architecture for Future Wireless Communications, Auerbach, CRC Press, Boca Raton, Fla, USA, 2007.

[9] A. Nosratinia, T. Hunter, and A. Hedayat, "Cooperative communication in wireless networks," IEEE Commun. Mag., vol. 42, no. 10, pp. 74-80, Oct. 2004.

[10] N. Levy, and S. Shamai (Shitz), "Information Theoretic Aspects of Users' Activity in a Wyner-Like Cellular Model," IEEE Trans. Inf. Theory, vol. 56, no. 5, pp.2241-2248, May, 2008.

[11] X. H. You, D. M. Wang, B. Sheng, X. Q. Gao, X. S. Zhao, and M. Chen, "Cooperative distributed antenna systems for mobile communications," IEEE Wireless Commun., vol. 17, no. 3, pp.35-43, June, 2010.

[12] S. Chatzinotas, M. A. Imran, and R. Hoshyar, "On the multicell processing capacity of the cellular MIMO uplink channel in correlated Rayleigh fading environment," IEEE Trans. Wireless Commun., vol. 8, no. 7, pp.3704-3715, July, 2009.

[13] G. Fraidenraich, O. Lvque, and J. M. Cioffi, "On the MIMO Channel Capacity for the Nakagami- Channel," IEEE Trans. Inf. Theory, vol. 54, no. 8, pp.3752-3757, Aug., 2008.

[14] N. C. Beaulieu, and C. Cheng, "Efficient Nakagami-m Fading Channel Simulation," IEEE Trans. Veh. Technol., vol. 54, no. 2, pp.413-424, Mar., 2005.

[15] D. Kaltakis, E. Katranaras, M.A. Imran, and C. Tzaras, "Information Theoretic Capacity of Gaussian Cellular Multiple-acess MIMO fading channel," IET Commun., vol. 3, no. 7, pp.1201-1207, 2009.

[16] A. D. Wyner, "Shannon-theoretic approach to a Gaussian cellular multiple-access channel," IEEE Trans. Inf. Theory, vol. 40, pp. 17131727, Nov. 1994.

[17] Ross S. M.: 'Introduction to probability models' (Academic Press, 2007, 9th edn.), pp. 64-73

[18] I. Gradshteyn and I. Ryzhik, "Table of integrals, series, and products," London, UK: Academic Press, 2003.

[19] S. Chen, W. Wang, and X. Zhang, "Asymptotic analysis of multiuser diversity and selection diversity in multiple-relay networks," in Proc. IEEE Int. Conf. Commun., pp. 1-5, 2009.

[20] H. A. Suraweera, M. Soysa, C. Tellambura, and H. K. Garg, "Performance analysis of partial relay selection with feedback delay," IEEE Signal Processing Letters, vol. 17, no. 6, pp.531-534, June, 2010. 